Ruiz, E. y Oviedo, J. (2021). Bases sociales para la configuración de un modelo de gestión financiera con perspectiva solidaria en el contexto de organizaciones rurales. Contaduría Universidad de Antioquia, 78, 155-177. Doi:

https://doi.org/10.17533/udea.rc.n78a06

\title{
Bases sociales para la configuración de un modelo de gestión financiera con perspectiva solidaria en el contexto de organizaciones rurales
}

Erika Yissela Ruiz Muñoz

Universidad del Cauca

eyissela@unicauca.edu.co

Orcid: 0000-0002-3018-1768

Juan Ignacio Oviedo Pino

Universidad del Cauca jioviedo@unicauca.edu.co

Orcid: 0000-0002-0548-9699 
Ruiz, E. y Oviedo, J. Bases sociales para la configuración de un modelo de gestión financiera...

\section{Bases sociales para la configuración de un modelo de gestión financiera con perspectiva solidaria en el contexto de organizaciones rurales}

Resumen: Las finanzas solidarias, a diferencia de las finanzas ortodoxas, se caracterizan por un enfoque social y relacional de los colectivos humanos; en esta línea, la presente investigación indaga sobre las bases sociales necesarias para la construcción de un modelo de gestión financiera congruente con el contexto de las comunidades rurales como las existentes en el departamento del Cauca (Colombia), atendiendo a sus experiencias de solidaridad y a los ejes de su realidad social y organizacional. Utilizando un enfoque mixto o triangulado, se fundamenta en metodologías de diagnóstico rural e investigación acción participativa. Como resultado se estructura una propuesta general que revaloriza los ejes de las denominadas finanzas solidarias.

Palabras clave: Economía social y solidaria, finanzas rurales, finanzas solidarias, gestión organizacional, contexto rural.

Social bases for the configuration of a financial management model under a solidarity perspective in the context of rural organizations

Abstract: Solidarity finance, unlike orthodox finance, is characterized by a social and relational approach of human collectives. In this line, the present research inquiries on the social bases needed for the construction of a financial management model consistent with the context of rural communities such as the ones existing in the Cauca state (Colombia), taking into account their solidarity experiences and the axes of their social and organizational reality. Using a mixed or triangulated approach, the work is founded on rural diagnostic methodologies and participative action research. As a result, a general proposal is structured which revalues the axes of the so-called solidarity finance.

Keywords: social and solidarity economy, rural finance, solidarity finance, organizational management, rural context

Bases sociais para a configuração de um modelo de gestão financeira com perspectiva solidária no contexto de organizações rurais

Resumo: As finanças solidárias, a diferença das finanças ortodoxas, estão caracterizadas por um enfoque social e relacional dos coletivos humanos; nesse sentido, esta pesquisa indaga sobre as bases sociais necessárias para a construção de um modelo de gestão financeira congruente com o contexto das comunidades rurais como as existentes no departamento do Cauca (Colombia), atendendo a suas experiências de solidariedade e aos eixos de sua realidade social e organizacional. Utilizando um método misto ou de triangulação, fundamenta-se em metodologias de diagnóstico rural e pesquisa ação participante. Como resultado estrutura-se uma proposta geral que revaloriza os eixos das denominadas finanças solidárias.

Palavras-chave: Economia social e solidária, finanças rurais, finanças solidárias, gestão organizacional, contexto rural

Des bases sociales pour la configuration d'un modèle de gestion financière avec une perspective solidaire dans le contexte des organisations rurales.

Résumé:

La finance solidaire, contrairement à la finance orthodoxe, est caractérisée par une approche sociale et relationnelle des collectifs humains. Dans ce sens, cette recherche étudie les bases sociales nécessaires à la construction d'un modèle de gestion financière congruent avec le contexte des communautés rurales telles que celles existant dans le département de Cauca en Colombie, tout en tenant compte de leurs expériences de solidarité et des axes de leur réalité sociale et organisationnelle. Au moyen de l'application d'une approche mixte ou triangulée, elle s'appuie sur des méthodologies de diagnostic rural et de recherche-action participative. En conséquence, une proposition générale est structurée, elle réévalue les axes de la soi-disant finance solidaire.

Mots clés: économie sociale et solidaire, finance rurale, finance solidaire, gestion des organisations, contexte rural. 


\title{
Bases sociales para la configuración de un modelo de gestión financiera con perspectiva solidaria en el contexto de organizaciones rurales
}

\author{
Erika Yissela Ruiz Muñoz y Juan Ignacio Oviedo Pino \\ https://doi.org/10.17533/udea.rc.n78a06 \\ Primera versión recibida en noviembre de 2020 - Versión aceptada en abril de 2021
}

\section{Introducción}

Como campo de conocimiento, las finanzas se han caracterizado por su orientación al manejo de recursos económicos en contextos de incertidumbre y riesgo con miras a alcanzar el mayor valor posible. Por ende, en el paradigma moderno se pueden encontrar vertientes como las finanzas corporativas y de mercado que han impuesto técnicas matemáticas y estadísticas como instrumentos adecuados de investigación, definiendo el papel economicista racional de los individuos, con un constructo teórico bajo un paradigma funcionalista y operativo.

Esta lógica racional ha venido siendo cuestionada de manera reciente, dando como resultado nuevas apuestas sobre la forma de tomar decisiones financieras, y posicionando la relevancia de estudiar el comportamiento humano, su complejidad y variabilidad. En efecto las finanzas como campo de conocimiento, sin abandonar en primera instancia su enfoque cuantitativo, se ha abierto a escenarios de mayor incertidumbre con la comprensión de las formas de vida de la gente, dando un importante espacio al estudio de las emociones y la cultura como elementos que afectan las decisiones individuales y colectivas, cobrando fuerza el campo de las finanzas del comportamiento (Nassar, 2019) y otras líneas con mayor o menor grado de heterodoxia, entre las que se pueden mencionar las finanzas populares, las finanzas rurales, las finanzas islámicas, las finanzas solidarias y las microfinanzas, las cuales comparten el hecho de ser visiones alternativas a la corriente principal y destacan el carácter social y relacional de los colectivos humanos. Como lo plantea Carrizo (2019) existen 
Ruiz, E. y Oviedo, J. Bases sociales para la configuración de un modelo de gestión financiera...

finanzas en otras economías que se contrapone al imaginario del sujeto económico racional:

Un sujeto social e interdependiente que puede aportar al sistema y no solamente recibir, es al mismo tiempo, una ruptura y un cuestionamiento al mensaje mediático que asocia el éxito al hombre económico racional y maximizador de beneficios, que actúa en un sistema económico de suma cero. Si una persona que ha sido exiliada de la ecuación de suma cero, puede reproducir la vida con dignidad, es una constatación de las diferencias, de la diversidad y de la alteridad en todas sus potencias. (...) La alteridad se usa aquí desde el lazo social, desde la idea de sociedad que prioriza a la persona y al trabajo, a la solidaridad y al conjunto, independientemente de las particularidades de cada uno. Se trata del conjunto que se liga entre otros que no necesariamente son iguales. (pp. 30-31)

Lo anterior, ha llevado a inferir que los modelos contables y financieros de la economía imperante no se circunscriben a organizaciones del mundo rural o a sectores populares (Acosta, 2013). Desde esta postura, se torna fundamental aprender de experiencias dadas en comunidades de base rural cuya gestión de recursos les ha permitido sobrevivir a un entorno de alta competitividad y exclusión. Acercarse a dichas experiencias se convierte en un medio legítimo de sentar bases para la construcción de un modelo de gestión financiera solidaria, acorde con el entorno y con mayores posibilidades de ser apropiable en diferentes entornos rurales, lo cual implica, en primera instancia, desligar las relaciones sociales y la contabilidad del reduccionismo económico-financiero y monetario (Mejía, 2020), debido a que en la economía popular y solidaria el dinero es considerado en un segundo plano (León, 2018).

En conexión con lo anterior, la presente investigación busca configurar las bases sociales, entendidas estas como el conglomerado de actores sobre el que se sustenta las relaciones humanas, económicas, políticas, sociales, ambientales, con rasgos distintivos poblacionales y de territorio, necesarias para la construcción futura de un modelo de gestión financiera que defiende el acceso y uso sustentable de los recursos, involucrando variables culturales y ambientales ${ }^{1}$, congruente con el contexto de las comunidades rurales como las existentes en una región particularmente marginada y azotada por la violencia, donde concurren poblaciones afros, indígenas y campesinos, entre otros, como es el departamento del Cauca (Colombia). Para esto, se realiza la investigación tomando como referencia las experiencias de cuatro organizaciones donde son evidentes

1 Esto se hace para emular el hecho que las finanzas convencionales se derivan de la vertiente económica neoclásica. Es por ello que las finanzas con perspectiva solidaria se plantean aquí como una rama de la denominada economía social y solidaria - ESS-, cuya novedad radica en "posicionar un discurso alternativo en materia de desarrollo humano y económico, fuertemente crítico con los resultados mostrados por el capitalismo neoliberal" (Guerra, 2010, p. 69). 
los procesos- asociativos autogestionados y pensados desde el bien común, las cuales fueron escogidas por su disposición a la presente investigación ${ }^{2}$.

Es un trabajo mixto o triangulado que articula diferentes fuentes teóricas, datos y métodos de investigación (Forni y De Grande, 2020), el cual parte de una visión agregada (departamental y municipal) para llegar a las organizaciones seleccionadas, con quienes son aplicadas herramientas cualitativas y en alguna medida cuantitativas; con esta metodología se logra evidenciar cómo en el departamento del Cauca han aplicado estrategias financieras acordes con el territorio, cultura, economía y población, y cuya caracterización es un insumo valioso para aproximarse a la definición de las bases de un modelo de gestión financiera en contextos rurales del mismo departamento y de la región.

Como complemento, se estructura una propuesta general que revaloriza los ejes de las denominadas finanzas solidarias, incorporando los insumos dados por las comunidades participantes de la investigación, y con lo cual se pretende contribuir a la construcción de una propuesta colectiva de gestión financiera en las comunidades rurales. Este tipo de ejercicio, sin la pretensión de construir un modelo genérico, deja algunos elementos de referencia para futuras investigaciones en el área de las finanzas solidarias en lo que respecta a su sinergia con las ciencias de la gestión y los estudios interdisciplinarios del desarrollo, además que brinda un resultado concreto donde la creación de conocimiento se hace de manera colaborativa con las comunidades rurales valorando sus saberes y experiencias.

\section{Alternativas analíticas para las finanzas en contextos rurales}

\section{II.1 De las finanzas corporativas a la visión solidaria de las finanzas}

Las finanzas en el sentido contemporáneo se fundamentan en el pensamiento de la escuela clásica europea en cabeza de Adam Smith, según la cual, la economía se orienta por principios individualistas, tendientes a la maximización de utilidades, constituyendo el carácter racional de los seres humanos (Landreth y Colander, 2006). Como rama de la economía (específicamente de la microeconomía), las finanzas convencionales se ocupan de la asignación de recursos escasos (Bodie y Merton, 2003). En esta dirección, las finanzas ortodoxas se han desarrollado en un marco pragmático que supone la generación de valor; en consecuencia, su alcance se ha visto acotado a tres conceptos fundamentales: presupuesto de capital, la estructura del capital y la administración del capital (Ross, Westerfield y Jaffe, 2012). Esta visión puramente financista ha alejado las

2 Las organizaciones rurales seleccionadas para el estudio se han visto beneficiadas por contribuciones de entidades públicas y privadas, entre las que se encuentra la Universidad del Cauca y la Federación Nacional de Cafeteros a través del Comité Departamental de Cafeteros del Cauca. 
Ruiz, E. y Oviedo, J. Bases sociales para la configuración de un modelo de gestión financiera...

finanzas tradicionales del medio social en que subsiste, individualizando los resultados y desdemocratizando los controles populares (Pérez, 2016).

Por fortuna, las finanzas como campo de conocimiento han venido ampliando su alcance y aplicación práctica, al pasar de la teoría de portafolios hacia niveles más complejos de estudio como las finanzas sociales y las comportamentales (Saavedra, M. L. y Saavedra M. J, 2012). Si bien el sistema económico contemporáneo favorece el desarrollo y estudio de las primeras, lo económico no puede estar por fuera de la naturaleza (Coraggio, 2011) y por lo tanto, es posible hablar de apuestas alternativas donde la impronta es el carácter social de las relaciones humanas; estas alternativas tienen su origen en la llamada economía social y solidaria, en tanto vertiente que coloca al ser humano en la base, "con la finalidad de ampliar y/o mejorar las condiciones de vida -sea de sus propios integrantes, de un sector específico de la sociedad, de la comunidad local y/o del entorno" (Chiroque y Niño, 2019, p. 250), y en donde se dirigen los objetivos económicos, sociales y financieros a la satisfacción de las necesidades humanas ${ }^{3}$.

Desde la perspectiva de la ampliación de la cobertura de los servicios financieros, las finanzas solidarias constituyen una adecuación de estos, estableciendo relaciones duraderas y sustentables de economía y sociedad (Altadill, 2015), las cuales provocan identificación y autorreconocimiento dentro de economías alternativas (Gibson-Graham, 2007). Ahora bien, se debe aclarar que las finanzas solidarias así concebidas no tienen una definición establecida en la literatura y generalmente aceptada por los estudiosos de la economía solidaria, pero existen algunas aproximaciones como la planteada por Quiñones y Fernando (2003, p. 4) en el marco del denominado Taller FinSol de abril de 2001 donde:

...[se] intentó definir las Finanzas Solidarias como «un sello»de finanzas éticas, responsables y sustentables que sólo podría ser aplicado a ciertos tipos de instituciones que respetan abordajes y métodos y [estarían constituidas para proveer] (...) servicios orientados al refuerzo de relaciones sociales y a la producción de capital social.

La economía de la solidaridad, como modo especial y distinto de hacer economía (Guerra, 2014), se inserta en el conjunto de propuestas epistemológicas que intentan analizar la sociedad en sus múltiples dimensiones. La economía social y solidaria es una respuesta a la afirmación del Foro Social Mundial de que otra economía es posible (Coraggio, 2011). Esta surge como una forma de reafirmar los procesos de inclusión social, donde se gesta el crecimiento de las relaciones sociales. En esta línea, la ESS es un espacio común a las experiencias históricas del cooperativismo y mutualismo (Pastore, 2010).

3 En esta dirección el economista Manfred Max-Neef propone "una comprensión de la estructura y dinámica de aquellos aspectos de las actividades sociales que denominamos «sistema económico» desde la perspectiva de la atención de las necesidades humanas básicas, incluidas dentro del marco social y ecológico" (Max Neef, 1998, p. 14). 


\section{II.2 Aproximación a las características de las finanzas solidarias en contextos rurales}

La economía social y solidaria en su conjunto, y en especial las finanzas solidarias como subrama, contribuyen en el ejercicio económico y financiero de las organizaciones del tercer sector, pero más en aquellas del sector rural, pues en ellas la gestión se realiza por medio del acuerdo colectivo de los integrantes de los grupos de trabajo u organizaciones basándose en la premisa de reciprocidad. Es así como desde el enfoque de las comunidades rurales, además de reconocer los elementos materiales necesarios para el crecimiento de la producción, el recurso principal son las personas, y es de ellas (y con ellas) donde surgen las iniciativas comunes.

En el marco de las alternativas analíticas sobre finanzas en el entorno rural, Nagarajan y Meyer $(2005$, p. 7$)$ indagan sobre las denominadas finanzas rurales ${ }^{4}$ en donde se considera "a las poblaciones de las áreas rurales como susceptibles de recibir servicios bancarios a través de instituciones eficaces". No obstante, es de anotar que, en la actualidad, a pesar del avance en las herramientas tecnológicas que contribuyen a llevar los servicios financieros a un número considerable de personas, aún existen limitantes para expandir los productos para los pobladores de zonas rurales en comparación con el financiamiento urbano.

De igual manera, otro referente conceptual son las finanzas populares, las cuales se inscriben en la diversidad y amplitud de ideas, proyectos y realidades que convergen en el planteamiento de crear y desarrollar propuestas y escenarios en torno a lo económico (Ortega, 2008), y que para el caso latinoamericano se representan a través de organizaciones de base, movimientos sociales, minorías, ONG, entre otros.

Finalmente, se encuentran las ideas principales de la teoría económica islámica - la lujuria de los bienes, el papel del dinero, la solidaridad, la justicia social que han derivado en principios generalmente aceptados para las llamadas finanzas islámicas —riba o interés, le gharar o incertidumbre, le maysir o especulación, acumulación de dinero, especulación, actividades ilegales- (Causse, 2012). Aquellas tienen lecciones para ser aprendidas por las finanzas convencionales, como quiera que estas por definición están fundamentadas en el individualismo.

Para puntualizar, Rafael Franco (2014), promotor de la contametría propone cuatro ejes de estudio para un mayor acercamiento de las finanzas a la sociedad, los cuales se acuñan en el presente trabajo, a saber: territorio, población, cultura y economía. Ejes que si se trabajan en conjunto logran un desarrollo social integral.

4 Siguiendo a Nagarajan y Meyer (2005, p. 1): "el término finanzas rurales engloba tanto las finanzas agrícolas como las microfinanzas rurales, y constituye un sub-sector del sector financiero global". 
Ruiz, E. y Oviedo, J. Bases sociales para la configuración de un modelo de gestión financiera...

El territorio, como eje central, puede ser concebido como un espacio o lugar delimitado donde una o varias comunidades realizan sus prácticas productivas, ambientales, económicas, políticas, culturales y de relacionamiento social, este territorio puede contener un entorno natural específico y varias identidades por las características propias de sus pobladores; es decir, un territorio no es simplemente el lugar, su distinción como territorio se compone por su biodiversidad, creencias, tradiciones e ideologías.

La cultura como eje ha sido invisibilizado a la luz de las finanzas, la cual, en una lectura amplia, implica tradiciones, hábitos, educación, valores, sistemas productivos — producción y distribución-, así como la conducta y normas.

La población se ha analizado, principalmente, desde sus funciones como fuerza de trabajo, capacidades de carga entre grupos productivos y no productivos, ocupación formal, desocupación, subempleo, calificación de la mano de obra, presión laboral en los salarios, etc., y en el campo financiero como grupo objetivo de políticas de colocación de créditos, mas no en una dimensión social y solidaria. La comunidad, como concepto asociado, es una dimensión inseparable de la especie humana (Peña, 2017) y en lo interno y en principio son solidarias (Coraggio, 2009).

El eje económico involucra normas, valores, prácticas e instituciones, que una comunidad voluntariamente instaura para organizar el "metabolismo seres humanos - naturaleza mediante actividades interdependientes", en el denominado ciclo económico (Coraggio, 2009, p. 286), y de esta manera satisfacer las necesidades legítimas de todos.

\section{II.3 Apuesta institucional sobre Economía y Finanzas Solidarias: el caso Colombia}

En Colombia la economía solidaria se considera un sector especial de la economía, con una normatividad diferenciada a la que reglamenta a las empresas de capital privado, por lo cual es de importancia exponer lo que nos propone la perspectiva institucional sobre el tema.

Se hace necesario poner de antecedente, la existencia, en los años noventa del siglo pasado, de un sector cooperativo financiero, de ahorro y crédito que atendía las necesidades de las clases populares colombianas, y que para 1997 estaba conformado por tres bancos cooperativos. En esta época, dicho sector se enfrentó a situaciones que lo llevaron a una crisis tendiente a su desaparición, en cuanto se atenuó la recesión económica del país y la crisis del sector financiero tradicional; así las organizaciones del sector solidario quedaron a merced del financiamiento que ofrece la banca tradicional.

En consecuencia, el Estado colombiano actualmente ha dispuesto la Unidad Administrativa Especial de Organizaciones Solidarias UAEOS pensada para el fomento y fortalecimiento de este tipo de organizaciones, entre las que se 
encuentran: cooperativas, fondos de empleados, asociaciones mutuales, corporaciones, fundaciones, grupos de voluntariados, entre otros. En esta línea, la legislación colombiana reconoce dos categorías en las que se distribuyen las organizaciones del sector solidario: "Organizaciones de Economía Solidaria" y las "Organizaciones Solidarias de Desarrollo"; aquí es donde se pueden distinguir las personas jurídicas que constituyen las organizaciones solidarias formales, que, además de tener deberes sociales, tienen obligaciones legales y tributarias, con algunas excepciones por el cumplimiento de sus propósitos sociales.

De igual manera, también se tiene como referente institucional en Colombia el reciente denominado Plan Nacional de Fomento a la Economía Solidaria y Cooperativa Rural PLANFES, en el que se establecen unos objetivos específicos en procura de contribuir con la paz, la equidad y la educación. Estos objetivos se orientan hacia el fortalecimiento y la promoción de la cultura asociativa solidaria, para la generación de ingresos, de trabajo decente, inclusión social, igualdad de oportunidades y construcción de tejido social, como fundamento de una Colombia en paz.

A diferencia de las microfinanzas, las cuales intentan imitar a las finanzas solidarias sin poder lograrlo (Chao-Beroff y Prébois, 2001), los objetivos estratégicos de las finanzas solidarias se agrupan en tres ejes funcionales: las capacidades de autogestión comunitaria y cultura solidaria, la autogestión para la generación de ingresos y las sinergias institucionales. Las finanzas solidarias se desarrollan en torno a la iniciativa de una comunidad, con el establecimiento de unos principios y valores que buscan fortalecer las condiciones sociales, económicas, culturales y ambientales, en tanto que trabajan desde la reciprocidad de las personas consigo mismas y con su entorno.

En consonancia con lo planteado, se pueden apuntar cuatro ideas generales que permiten dar cierre al presente apartado:

1. El estudio de las finanzas contemporáneas es hoy más amplio, de modo tal que apuestas como las finanzas solidarias vienen cobrando una importancia relativa al diferenciarse de las finanzas corporativas en su objeto, enfoque, ejes, grupos de interés y escenarios de actuación.

2. Las finanzas solidarias son una alternativa analítica pertinente para los escenarios rurales que, si bien comparte planteamientos similares con las finanzas rurales, finanzas populares e islámicas, tiene unos atributos particulares derivados del cuerpo teórico provisto por la economía social y solidaria, en el cual tienen espacio las variables económicas del pensamiento convencional junto a otras de carácter alternativo.

3. Los estudios sobre finanzas solidarias deben sin duda involucrar elementos de la teoría financiera — eje económico-, de la sociología y antropología - eje poblacional y cultural- y de las ciencias naturales — eje territorial-, es decir, una aproximación interdisciplinaria, en tanto las finanzas 
Ruiz, E. y Oviedo, J. Bases sociales para la configuración de un modelo de gestión financiera...

ortodoxas por sí solas dejan por fuera las variables sociales y del entorno al momento de tomar decisiones para la gestión financiera organizacional.

4. Para el caso colombiano, la revisión normativa institucional al sector de la economía solidaria y sus diversas prácticas, resulta importante para identificar los puntos diferenciadores de las finanzas solidarias ante las microfinanzas, en tanto estas últimas se enfocan en la maximización de utilidades por medio de la expansión de servicios financieros accesibles al tener menos exigencias que la banca convencional —incorporando solamente el eje económico-.

\section{Caracterización y diagnóstico}

Esta investigación se sustenta en la aplicación de las metodologías de Diagnóstico Rural Participativo (Chambers, 1997) e Investigación Acción Participativa (Pérez, Maya y Farra, 2001), en la que los objetivos son conocidos tanto por los investigadores como por la comunidad, promoviendo la construcción conjunta de conocimiento (Contreras, 2002). Entre las aproximaciones metodológicas se realizaron talleres grupales para la sistematización de experiencias con los miembros de las organizaciones rurales (FAO, 2012; Jara, 1994) y entrevistas individuales a profundidad con la(s) persona(s) encargada(s) de la información financiera. Se creó un instrumento híbrido que sintetiza la ficha de caracterización de competencias organizacionales usada para la elaboración de estrategias de inclusión productiva (Rivas, 2013), y el formato de encuesta para el análisis de condiciones financieras de organizaciones del sector agro en el Cauca (Díaz, 2016).

El ejercicio investigativo se llevó a cabo a partir de las actividades que se muestran a continuación:

a) Diseño de un instrumento metodológico

b) Acercamiento a experiencias organizacionales del sector rural

c) Caracterización de organizaciones rurales

d) Socialización / validación del ejercicio de caracterización y construcción de bases del modelo

e) Consolidación de los hallazgos

Con fundamento en la metodología, para el desarrollo de la segunda actividad, se focalizaron organizaciones del centro, nororiente y sur caucano, a saber: la Asociación de Prosumidores Agroecológicos Agrosolidaria Seccional Piagúa (municipio de El Tambo - zona centro), Asociación de Productores Agropecuarios de Bolívar ASPABOL (municipio de Bolívar - zona sur), la Asociación de Productores Cristianos de Café Especial del Oriente Caucano y Occidente del Huila ASPROFECH (Municipio de Páez - zona nororiente), y Enredarte con Identidad (municipios de Corinto, Caldono, Jambaló, Toribío y Silvia -zona nororiente). Estas organizaciones fueron seleccionadas, además, considerando su ubicación, 
su trayectoria —etapas de éxito y obstáculos-, dinámicas productivas —actividades diferenciadas entre ellas-, impacto generado en su entorno - con beneficios a la comunidad-, su estructura organizacional, así como la filosofía y consigna organizacional enfocada al buen vivir, como seguidamente se aprecia.

Agrosolidaria Seccional Piagúa se constituye formalmente en el año 2010, logrando canalizar recursos de ayudas externas y apoyar procesos de fortalecimiento de la cadena de aguacate y en especies menores. ASPABOL es una organización formalizada en el año 1998, creada por agricultores, especialmente cultivadores de caña panelera. ASPROFECH es una iniciativa que se originó en la vereda San Luis al sur del municipio de Páez en octubre del 2012, como una reacción de la comunidad ante los bajos precios del café; la organización ha tenido gran acogida por la comunidad incrementando con el tiempo el número de asociados. Enredarte con identidad es una red de artesanas que reúne a Kumbiaxca de Corinto, Filigranas de Caldono, Backuwe de Jambaló, Waka't kiwe de Toribío y de Manos Silvianas del municipio de Silvia. La red se originó en el año 2011 procurando que el trabajo de estos grupos de artesanas sea valorado, y el arte del tejido no se pierda dentro de las culturas.

Algunas características demográficas se presentan en las Tablas 1 a 6 .

Tabla 1: Características constitutivas de los hogares de los asociados de las organizaciones caracterizadas

\begin{tabular}{|l|c|c|c|c|}
\hline Estadísticos descriptivos de los hogares & Agrosolidaria Piagúa & ASPABOL & ASPROFECH & Enredarte \\
\hline Mujeres & $57,14 \%$ & $40 \%$ & $7,14 \%$ & $90 \%$ \\
\hline Hombres & $42,86 \%$ & $60 \%$ & $92,86 \%$ & $10 \%$ \\
\hline Edad promedio general & 50,7 & 46 & 39,75 & 45,50 \\
\hline Edad promedio mujeres & 50 & 43 & 54 & 46,44 \\
\hline Edad promedio hombres & 51 & 50 & 39,75 & 37 \\
\hline Tenencia de la finca (años promedio) & 19 & 42 & 14,6 & 19,8 \\
\hline Tamaño de hogar (promedio) & 5 & 4,5 & 3,5 & 4,8 \\
\hline \% de menores en hogares & $16,67 \%$ & $20,90 \%$ & $30,43 \%$ & $22,92 \%$ \\
\hline Hogares con un solo aportante & $0 \%$ & $46,67 \%$ & $38,46 \%$ & $20 \%$ \\
\hline
\end{tabular}

Fuente: Elaboración propia con base a información de las organizaciones caracterizadas. 
Ruiz, E. y Oviedo, J. Bases sociales para la configuración de un modelo de gestión financiera...

Tabla 2: Participación de los asociados en prácticas solidarias

\begin{tabular}{|l|c|c|c|c|}
\hline \multicolumn{1}{|c|}{ Participación en prácticas solidarias } & Agrosolidaria Piagúa & ASPABOL & ASPROFECH & Enredarte \\
\hline 1. Bingo & $14,29 \%$ & $46,67 \%$ & $30,77 \%$ & $30 \%$ \\
\hline 2. Rifa & $42,86 \%$ & $73,33 \%$ & $53,85 \%$ & $60 \%$ \\
\hline 3. Préstamos sin interés & $42,86 \%$ & $73,33 \%$ & $69,23 \%$ & $10 \%$ \\
\hline 4. Colectas & $28,57 \%$ & $46,67 \%$ & $53,85 \%$ & $50 \%$ \\
\hline 5. Venta de alimentos & $28,57 \%$ & $53,33 \%$ & $46,15 \%$ & $60 \%$ \\
\hline 6. Donaciones & $28,57 \%$ & $60 \%$ & $61,54 \%$ & $50 \%$ \\
\hline 7. Arreglos de vías & $85,71 \%$ & $80 \%$ & $92,31 \%$ & $50 \%$ \\
\hline 8. Siembra de arboles & $57,14 \%$ & $86,67 \%$ & $76,92 \%$ & $50 \%$ \\
\hline 9.Festividades & $42,86 \%$ & $46,67 \%$ & $23,08 \%$ & $30 \%$ \\
\hline 10. Fiestas familiares & $57,14 \%$ & $93,33 \%$ & $84,62 \%$ & $30 \%$ \\
\hline 11.Cuidado de semillas & $42,86 \%$ & $46,67 \%$ & $30,77 \%$ & $40 \%$ \\
\hline 12. Recuperación de prácticas tradicionales & $28,57 \%$ & $33,33 \%$ & $46,15 \%$ & $30 \%$ \\
\hline 13. Mingas & $100 \%$ & $93,33 \%$ & $76,92 \%$ & $50 \%$ \\
\hline 14. Otro & $28,57 \%$ & $6,67 \%$ & $0 \%$ & $0 \%$ \\
\hline
\end{tabular}

Fuente: Elaboración propia con base en las organizaciones caracterizadas.

Tabla 3: Acceso a la tierra asociados de las organizaciones rurales caracterizadas

\begin{tabular}{|l|c|c|c|c|}
\hline $\begin{array}{c}\text { Distribución de las fincas por extensión } \\
\text { por hectáreas (ha) }\end{array}$ & Agrosolidaria Piagúa & ASPABOL & ASPROFECH & Enredarte \\
\hline Menor o igual a 0,5 ha & $14,29 \%$ & $6,67 \%$ & $7,69 \%$ & $40 \%$ \\
\hline Mayor a 0,5 y menor igual a 2 ha & $0 \%$ & $13,33 \%$ & $46,15 \%$ & $10 \%$ \\
\hline Mayor a 2 y menor igual a 5 ha & $42,86 \%$ & $46,67 \%$ & $23,08 \%$ & $40 \%$ \\
\hline Mayor a 5 y menor igual a 8 ha & $0 \%$ & $20 \%$ & $15,38 \%$ & $0 \%$ \\
\hline Mayor a 8 ha & $42,86 \%$ & $13,33 \%$ & $7,69 \%$ & $10 \%$ \\
\hline
\end{tabular}

Fuente: Elaboración propia con base a información de las organizaciones caracterizadas.

Tabla 4: Tipo de mano de obra utilizada por los hogares caracterizados

\begin{tabular}{|l|c|c|c|c|}
\hline \multicolumn{1}{|c|}{ Fuentes } & Agrosolidaria Piagúa & ASPABOL & ASPROFECH & Enredarte \\
\hline Jornalero & $71,43 \%$ & $73,33 \%$ & $100 \%$ & $20 \%$ \\
\hline Familia & $42,86 \%$ & $60 \%$ & $53,85 \%$ & $40 \%$ \\
\hline Mano prestada & $28,57 \%$ & $60 \%$ & $15,38 \%$ & $40 \%$ \\
\hline
\end{tabular}

Fuente: Elaboración propia con base a información de las organizaciones caracterizadas. 
Tabla 5: Fuentes de financiamiento

\begin{tabular}{|l|c|c|c|c|}
\hline Fuentes de Financiamiento / \% Hogares & Agrosolidaria Piagúa & ASPABOL & ASPROFECH & Enredarte \\
\hline 1. Amigos, vecinos, familia & $14,3 \%$ & $20 \%$ & $38,5 \%$ & $40 \%$ \\
\hline 2. Tendero & $42,9 \%$ & $20 \%$ & $23,1 \%$ & $10 \%$ \\
\hline 3. Bancos & $71,4 \%$ & $80 \%$ & $61,5 \%$ & $20 \%$ \\
\hline 4. Prestamistas & $14,3 \%$ & $13,3 \%$ & $30,8 \%$ & $0 \%$ \\
\hline 5. Organización donde trabaja & $28,6 \%$ & $0 \%$ & $15,4 \%$ & $0 \%$ \\
\hline 6. Prenderías & $0 \%$ & $0 \%$ & $0 \%$ & $0 \%$ \\
\hline 7. Cooperativas & $14,3 \%$ & $6,7 \%$ & $0 \%$ & $0 \%$ \\
\hline 8. Proveedores & $0 \%$ & $0 \%$ & $0 \%$ & $0 \%$ \\
\hline 9. Fundación - ONG & $0 \%$ & $0 \%$ & $0 \%$ & $0 \%$ \\
\hline 10. Cabildo & $0 \%$ & $0 \%$ & $0 \%$ & $0 \%$ \\
\hline 11. Caja de compensación & $0 \%$ & $0 \%$ & $0 \%$ & $0 \%$ \\
\hline 12. Recursos propios & $0 \%$ & $13,3 \%$ & $0 \%$ & $0 \%$ \\
\hline 13. Tarjeta de crédito & $0 \%$ & $0 \%$ & $0 \%$ & $0 \%$ \\
\hline Otro & $28,6 \%$ & $0,1 \%$ & $0 \%$ & $10 \%$ \\
\hline
\end{tabular}

Fuente: Elaboración propia con base en las organizaciones caracterizadas.

Tabla 6: Motivos de solicitud de financiamiento de las familias de las organizaciones caracterizadas

\begin{tabular}{|l|l|l|l|}
\hline \multicolumn{1}{|c|}{ Agrosolidaria Piagúa } & \multicolumn{1}{|c|}{ ASPABOL } & \multicolumn{1}{c|}{ ASPROFECH } & \multicolumn{1}{c|}{ Enredarte } \\
\hline Alimentación - Consumo & Alimentación & Alimentación & Alimentación \\
Pago trabajadores & Cultivo de caña & Abonos e Insumos & Cultivo de pollos \\
Vivienda & Cultivo de café & Gastos personales & Educación \\
Infraestructura & Cultivo de banano & Educación & Salud \\
Siembra de aguacate & Ganado & Negocio & Tienda \\
Educación & Mantenimiento de finca & Siembra de café & Arreglos de vivienda \\
Abonos & Abonos & Sostenimiento de la finca & \\
Maquinaria & Compra de terrenos & Replantación de café & \\
& & Pago trabajadores & \\
\hline
\end{tabular}

Fuente: Elaboración propia en base a información de las organizaciones caracterizadas.

Atendiendo al tipo de investigación, la presente tiene diferentes matices, tal como se aprecia en la Tabla 7, que permite un análisis integral de las diferentes variables de estudio. 
Ruiz, E. y Oviedo, J. Bases sociales para la configuración de un modelo de gestión financiera...

Tabla 7: Tipos de investigación de acuerdo con las ciencias sociales

\begin{tabular}{|l|l|}
\hline \multicolumn{1}{|c|}{ Criterio } & \multicolumn{1}{c|}{ Tipo de investigación y argumento } \\
\hline Método & $\begin{array}{l}\text { Investigación Inductiva: Partiendo de la observación de las experiencias } \\
\text { organizacionales, se realiza una comparación del quehacer organizacional } \\
\text { con los planteamientos teóricos ortodoxos y alternativos sobre las finanzas, } \\
\text { donde se descubren elementos comunes que dan apertura a un enfoque } \\
\text { general, para proponer un modelo de gestión financiera aplicable a } \\
\text { organizaciones de base rural. }\end{array}$ \\
\hline Propósito o finalidad & $\begin{array}{l}\text { Investigación aplicada: Esta investigación se logra con el acercamiento } \\
\text { a las comunidades rurales que permiten observar y registrar sus prácticas } \\
\text { organizativas. }\end{array}$ \\
\hline $\begin{array}{l}\text { Medios utilizados } \\
\text { para obtener los } \\
\text { datos }\end{array}$ & $\begin{array}{l}\text { Investigación de campo: Para desarrollar esta investigación se estructuran } \\
\text { talleres participativos con el fin de conocer las prácticas organizativas, } \\
\text { productivas y sociales. Se estructuran y utilizan dos formatos de encuesta } \\
\text { para caracterizar los hogares y la estructura organizacional. }\end{array}$ \\
\hline $\begin{array}{l}\text { Nivel de } \\
\text { conocimientos } \\
\text { adquiridos }\end{array}$ & $\begin{array}{l}\text { Investigación descriptiva: Por medio de la observación de las prácticas } \\
\text { organizativas y la caracterización se busca mostrar que elementos de } \\
\text { las finanzas convencionales y de las finanzas solidarias se tienen en las } \\
\text { organizaciones, con el propósito de reconocer las experiencias de las finanzas } \\
\text { en los entornos rurales. }\end{array}$ \\
\hline
\end{tabular}

Fuente: Adaptado de Marín (2008).

Con el fin de garantizar la coherencia entre las aproximaciones teóricas de la investigación, las herramientas metodológicas aquí usadas se encuentran orientadas en su mayoría a un enfoque cualitativo donde tienen lugar la participación, al habla y la escucha, la escritura e incluso el dibujo, con el fin de poder conocer los pensamientos y observar las diferentes prácticas inherentes a las organizaciones analizadas. Asimismo, con la intención de contrastar la teoría y las prácticas, así como identificar las bases sociales para la gestión financiera de estos modelos organizacionales, se contemplaron talleres grupales que retoman aspectos de la sistematización de experiencias con los miembros de las organizaciones rurales (Jara, 1994; FAO, 2012) sin llegar a ser una sistematización en sí misma.

Los encuentros planteados fueron: 1. Seminario-taller de introducción a las finanzas solidarias; 2 . Taller participativo de aplicación de instrumentos para la caracterización de las condiciones económicas de los asociados y la estructura organizacional; y 3 . Taller cualitativo de socialización y construcción de un plan de acción. En el Esquema 1 se resumen las etapas de investigación. 
Esquema 1: Etapas de la investigación

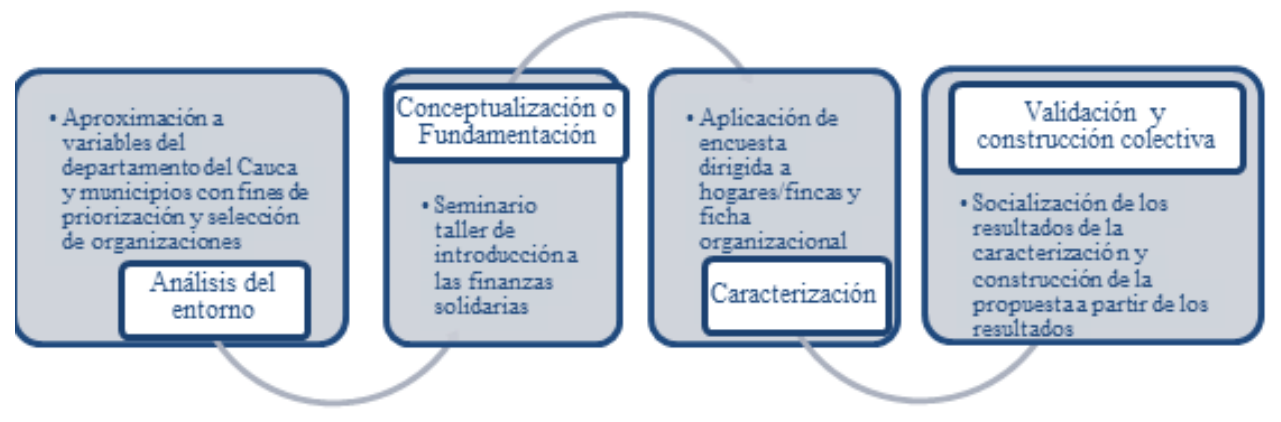

Fuente: Elaboración propia.

\section{Resultados y discusión}

Los resultados se dan desde la mirada de las organizaciones rurales participantes en la investigación, como protagonistas propias del estudio, en donde se evidencia la integración de los ejes constitutivos de la sociedad en la gestión organizacional.

Los asociados a Agrosolidaria Seccional Piagúa, en relación al eje medio ambiental, proponen: el mantenimiento y cuidado de bosques, la producción agroecológica, la reforestación, la identificación de zonas y especies de cuidado, la elaboración de un proyecto ambiental, la autogestión con recursos propios y donación de semillas; respecto a la cultura consideran la organización comunitaria de eventos; en la vía comunitaria, el fortalecimiento del lote colectivo que tienen destinado para la producción; y desde la perspectiva económica, proyectan ampliar la comercialización de las especies menores, mejorar la organización administrativa de la tienda comunitaria y la constitución de un almacén de agroinsumos.

Los asociados a ASPABOL contemplan la capacidad de otorgar préstamos a los asociados, gestionar la presentación de proyectos de vivienda, mejorar las condiciones y medios de vida, promover las capacitaciones, innovar en la producción y mejorar la comercialización de sus productos.

ASPROFECH, con el propósito de evolucionar su comercialización, se propone - además de seguir vendiendo café pergamino- tostar café especial para ofrecerlo molido y tener una marca propia para el abastecimiento local. Desde el enfoque administrativo, ven la necesidad de asignar cargos y responsabilidades con el reconocimiento de remuneraciones o bonificaciones, así como de definir capacitaciones para tener el conocimiento sobre presentación de propuestas/proyectos, y fortalecer los saberes técnicos productivos en la agricultura -o tener un agrónomo permanente en la organización para el estudio de los suelos y variedades de café-. En relación con lo económico, buscarán 
Ruiz, E. y Oviedo, J. Bases sociales para la configuración de un modelo de gestión financiera...

fortalecer el fondo rotatorio con el establecimiento de lineamientos sobre los créditos asociativos y los beneficios por el pronto pago para asociados.

En cuanto a Enredarte con Identidad, desde su visión holística consideran las siguientes acciones, a) respecto al eje económico, el intercambio de conocimientos/saberes con comunidades u organizaciones externas - por medio de jornadas de capacitación que la organización puede ofrecer-, la organización de un trueque, la producción de material propio - como la lana de ovejo y fique-, la vinculación de otras actividades productivas como la cerámica, costura, carpintería, forja, bisutería y bordado, asimismo, ampliar o tener mayor acceso a vitrinas para la exposición de sus productos en lugares estratégicos; b) desde el enfoque cultural, se proponen integrar otras técnicas de tejido e identificar la creación de cadena de valor, en cuanto se pueden realizar intercambios de conocimientos en tinturas, agujas y subproductos, crear escuelas de tejido para mujeres, jóvenes y niños.

En consonancia con lo expuesto por las organizaciones, la gestión debe pensarse con estrategias y compromisos colectivos que involucren variables para la protección social y ambiental, promuevan el trabajo decente, la inclusión social y la generación de riqueza comunitaria — representado en capital social- haciendo frente a las condiciones del mercado para un desarrollo sostenible en la ruralidad. Asimismo, el impacto de los planes de trabajo se debe medir desde los planteamientos de Coraggio (2013), quien define que las actividades deben ser:

...evaluadas no en términos de la máxima rentabilidad del capital [monetario] sino de: (i) su eficacia para producir a fin de resolver necesidades prioritarias y/o contribuir al mejor funcionamiento del sistema económico social y solidario en su conjunto, y de (ii) su efecto positivo directo e indirecto sobre las relaciones sociales y el contexto natural. (p. 36)

A su vez, en el marco de un plan de trabajo o modelo de gestión financiera se deben establecer unos procesos y estructura, de modo que se dé un orden a las actividades para conseguir los objetivos.

Considerando las organizaciones rurales, la gestión financiera en sus diferentes etapas debe complementar el trabajo comunitario que da valor agregado a la gestión y es más que pertinente incorporar los recursos no monetarios que el territorio le ofrece a la organización para cumplir sus propósitos. En este sentido, la propuesta contiene elementos que integran procedimientos y técnicas organizacionales e incluye las siguientes variables: i) objetivos organizacionales, ii) realidad social de la comunidad, iii) partes interesadas, iv) impacto territorial medio ambiental, v) asignación de cargos y responsabilidades, vi) apoyo técnico, tecnológico y profesional, vii) presupuestos, viii) soporte tecnológico, ix) definición de la producción, $\mathrm{x}$ ) definición de aportes financieros y no financieros, $\mathrm{xi}$ ) expectativa de beneficios, xii) inversiones, entre otros. 
El Esquema 2 representa las etapas de la gestión financiera de una organización rural, en donde la participación comunitaria está presente en cada etapa.

Esquema 2: Etapas de la gestión financiera en organizaciones de base rural

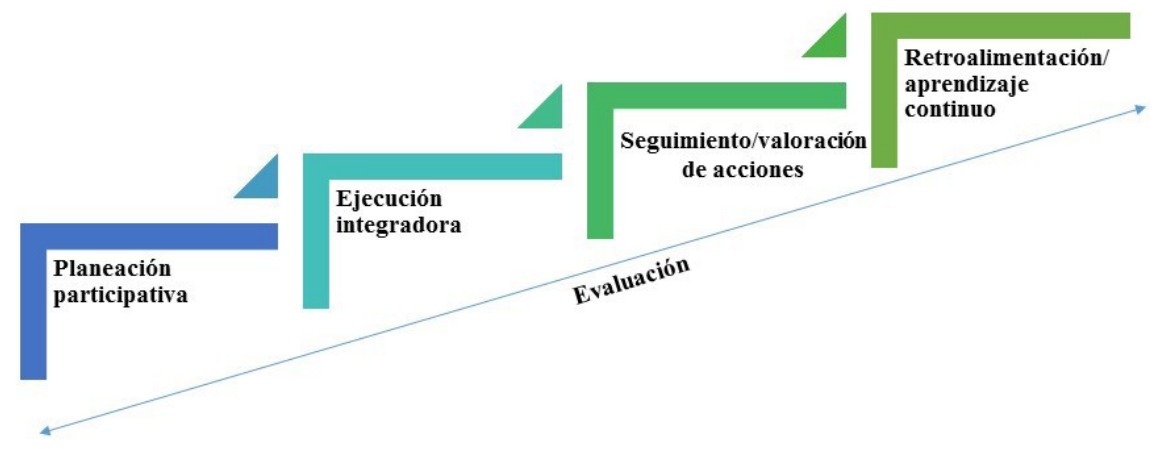

Fuente: Elaboración propia.

Los elementos de las finanzas solidarias están presentes en las cuatro etapas del ciclo por medio de los ejes cultural, territorial, económico y poblacional, en tanto la gestión estaría sobre los valiosos - muchos o pocos- recursos monetarios y no monetarios con que cuenta la comunidad, alineados con la sostenibilidad, la justicia social y estrategias de participación inclusiva (Sanchis y Campos, 2018).

En la primera etapa, la planeación se enfrenta a: 1) Establecer estrategias de participación colectiva y democrática como las asambleas generales y comités de trabajo que impulsen las decisiones organizacionales apropiadas para la comunidad —esto respondería al eje poblacional—; 2) Proyectar los ingresos y egresos siendo prudentes con la proyección de estos, para no sobrevalorar los rubros de ingresos y sin subestimar los gastos y costos de la organización, además de considerar previsiones por los riesgos ambientales a los que están expuestos los cultivos -que tendrían implicaciones en el eje económico-; 3) Potencializar el trabajo de los líderes y lideresas que conocen las particularidades, costumbres y creencias de la comunidad — todo esto como elementos de la cultura-; 4) Identificar los recursos que el ecosistema le ofrece a la comunidad y que la organización requiere utilizar para las actividades organizacionales, y establecer las acciones de cuidado que se deben tener en cuenta para mantener las riquezas naturales.

En la etapa de ejecución integradora es importante reconocer las habilidades de las personas para asignar responsabilidades coherentes, con un plan de trabajo bien definido que permita identificar los logros, y la supervisión de forma permanente por parte del colectivo. Además, el papel de las familias y de la comunidad hace viable o no las acciones, por lo que se necesita la voluntad 
Ruiz, E. y Oviedo, J. Bases sociales para la configuración de un modelo de gestión financiera...

y disposición para el logro de las metas. En la ejecución se debe reconocer a las familias como unidad de producción y de consumo, entendiendo que sus decisiones económicas no siguen la lógica del mercado (Gómez, 2000). De igual manera, las dinámicas del mundo moderno han llegado a las áreas rurales como instrumentos de soporte y comunicación, y cada vez más la educación formal se expande en los diferentes escenarios, y esto también influye en la gestión financiera de las organizaciones de base rural.

La etapa de seguimiento se puede hacer no solo como un ejercicio de verificación de lo ejecutado y logrado, sino también como un ejercicio que, articulado con la programación de actividades o plan de trabajo, permite evidenciar el nivel de avance por medio de los indicadores, revisar si las estrategias adaptadas permiten el logro de las metas o se deben reformular en el transcurso del plan de trabajo. En cuanto a las organizaciones de base rural se pueden determinar desde la planeación unos conductos regulares que den orden y faciliten el seguimiento tanto en lo productivo como en la organización, teniendo algunas consideraciones en diferentes niveles, tal como se muestra en el Esquema 3.

Esquema 3: Seguimiento del plan de trabajo desde las actividades organizacionales

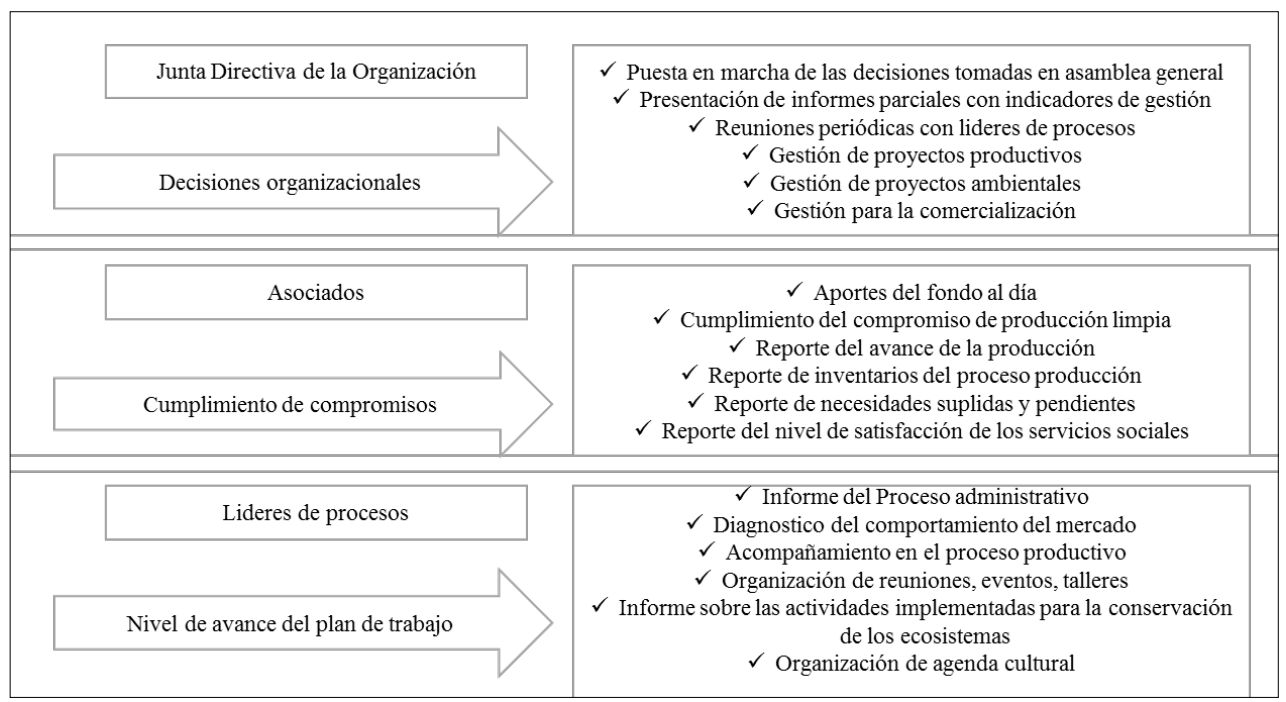

Fuente: elaboración propia.

La etapa de retroalimentación se considera fuente de nuevas ideas desde lo poblacional, territorial, cultural y económico, como se puede ver en Esquema 4. Las experiencias que las organizaciones puedan llegar a tener se deben tomar como aprendizajes que fortalezcan las iniciativas; esta fase final de reinicio procura identificar el impacto social/comunitario, cultural, económico y medio ambiental que las actividades organizacionales propiciaron. Esta revisión es el 
punto de partida de una nueva ruta de trabajo donde se retoman los aspectos positivos para fortalecerlos, y mantiene presente los desaciertos para no volver a llegar a ellos y hacerles frente a las nuevas condiciones del entorno.

Esquema 4: Frentes de aprendizaje continuo en las organizaciones de base rural

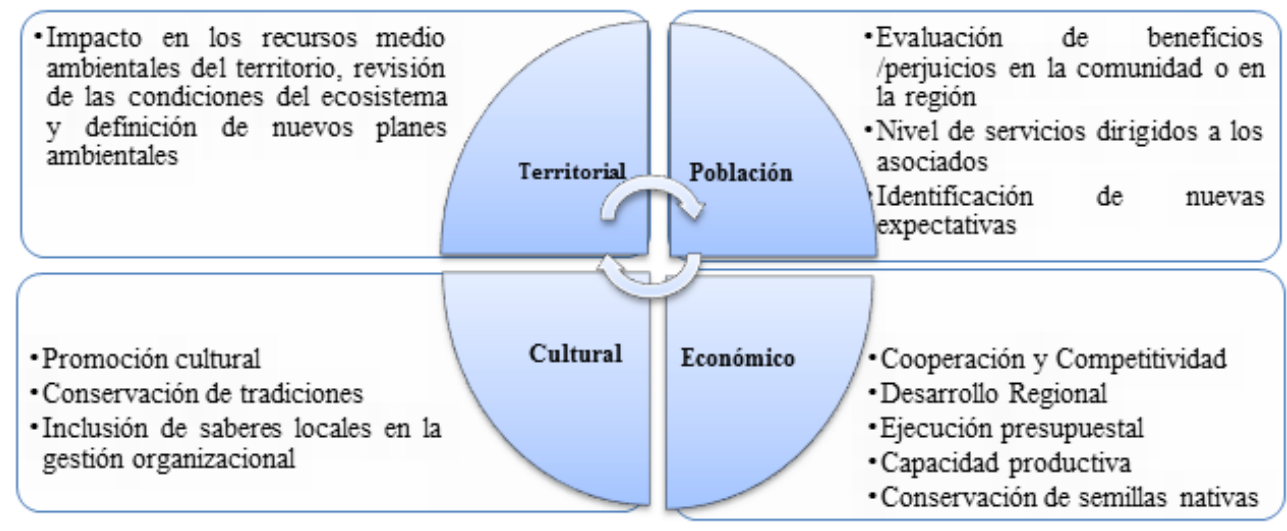

Fuente: Elaboración propia.

En la Tabla 8 se presenta un esquema en el cual se intenta concretar los elementos más significativos desde los ejes de las finanzas solidarias en relación con la gestión financiera.

Los hallazgos permiten resaltar la importancia de la confluencia de los saberes o conocimientos locales y los constructos teóricos prácticos de nivel global, y que estos elementos unidos favorecerían de manera positiva el quehacer de las organizaciones de base rural, donde el ser humano y sus expresiones sociales - con reciprocidad y solidaridad — dinamizan el día a día.

Tabla 8: Propuesta de variables a considerar en la gestión financiera con perspectiva solidaria en la ruralidad

\begin{tabular}{|c|l|l|l|l|}
\hline $\begin{array}{c}\text { Ejes/ Elementos } \\
\text { de la Gestión } \\
\text { Financiera }\end{array}$ & \multicolumn{1}{|c|}{$\begin{array}{c}\text { Dirección } \\
\text { Organizacional }\end{array}$} & $\begin{array}{c}\text { Implementación de } \\
\text { Políticas }\end{array}$ & $\begin{array}{l}\text { Administración de } \\
\text { los recursos }\end{array}$ & \multicolumn{1}{c|}{$\begin{array}{c}\text { Propósitos y/o } \\
\text { intereses }\end{array}$} \\
\hline Poblacional & $\begin{array}{l}\text { Se escucha la } \\
\text { opinión de todos } \\
\text { los asociados en } \\
\text { escenarios de } \\
\text { discusión colectiva, } \\
\text { en donde se toman } \\
\text { las decisiones en } \\
\text { consenso. }\end{array}$ & $\begin{array}{l}\text { Las políticas que se } \\
\text { implementan responden } \\
\text { a las requerimientos y } \\
\text { obligaciones que los } \\
\text { asociados han adquirido } \\
\text { con la organización } \\
\text { en relación con } \\
\text { lo productivo y lo } \\
\text { administrativo, } \\
\text { Reconocimiento de los } \\
\text { grupos etarios. }\end{array}$ & $\begin{array}{l}\text { Son los mismos } \\
\text { asociados los que } \\
\text { integran al grupo } \\
\text { que realiza estas } \\
\text { actividades a } \\
\text { través de cargos } \\
\text { como: presidentes } \\
\text { de asambleas, } \\
\text { secretarios, } \\
\text { tesoreros, y veedores. } \\
\text { Líderes de procesos. }\end{array}$ & $\begin{array}{l}\text { Las actividades que } \\
\text { la organización } \\
\text { realiza están } \\
\text { pensadas para } \\
\text { generar beneficios } \\
\text { colectivos en pro de } \\
\text { mejorar la calidad } \\
\text { de vida (1. Nivel de } \\
\text { vida; 2. Condiciones } \\
\text { de vida; 3. Medios } \\
\text { de vida). }\end{array}$ \\
\hline
\end{tabular}


Ruiz, E. y Oviedo, J. Bases sociales para la configuración de un modelo de gestión financiera...

\begin{tabular}{|c|c|c|c|c|}
\hline $\begin{array}{l}\text { Ejes/ Elementos } \\
\text { de la Gestión } \\
\text { Financiera }\end{array}$ & $\begin{array}{c}\text { Dirección } \\
\text { Organizacional }\end{array}$ & $\begin{array}{l}\text { Implementación de } \\
\text { Políticas }\end{array}$ & $\begin{array}{l}\text { Administración de } \\
\text { los recursos }\end{array}$ & $\begin{array}{l}\text { Propósitos y/o } \\
\text { intereses }\end{array}$ \\
\hline Económico & $\begin{array}{l}\text { La organización } \\
\text { prioriza los } \\
\text { cultivos que } \\
\text { contribuyan a } \\
\text { conseguir recursos } \\
\text { para invertir en } \\
\text { soporte para la } \\
\text { producción o } \\
\text { comercialización. }\end{array}$ & $\begin{array}{l}\text { - Constitución de } \\
\text { fondos de ahorro y } \\
\text { crédito } \\
\text { - Políticas de inversión } \\
\text {-Políticas de } \\
\text { financiamiento } \\
\text { - Políticas de inventarios }\end{array}$ & $\begin{array}{l}\text { Reinversión interna } \\
\text { de excedentes. } \\
\text { Se gestionan } \\
\text { proyectos para el } \\
\text { financiamiento } \\
\text { de propuestas } \\
\text { productivas. }\end{array}$ & $\begin{array}{l}\text { Lograr que las } \\
\text { actividades } \\
\text { productivas y } \\
\text { comerciales } \\
\text { generen excedentes } \\
\text { para retribuir en } \\
\text { beneficios para los } \\
\text { asociados. }\end{array}$ \\
\hline Territorial & $\begin{array}{l}\text { La organización } \\
\text { fomenta el } \\
\text { respeto con el } \\
\text { medio ambiente } \\
\text { y ecosistemas } \\
\text { específicos de los } \\
\text { territorios. }\end{array}$ & $\begin{array}{l}\text { Establecimiento } \\
\text { de políticas de } \\
\text { reforestación, de } \\
\text { cuidado de los } \\
\text { nacimientos de agua, y } \\
\text { de manejo de residuos. }\end{array}$ & $\begin{array}{l}\text { Se reconocen } \\
\text { las condiciones } \\
\text { ambientales de los } \\
\text { territorios para } \\
\text { definir cuál es el } \\
\text { mejor tratamiento y } \\
\text { cultivos para invertir. }\end{array}$ & $\begin{array}{l}\text { Cuidar la naturaleza } \\
\text { que provee } \\
\text { los espacios y } \\
\text { condiciones donde } \\
\text { se desarrollan las } \\
\text { actividades de la } \\
\text { organización. }\end{array}$ \\
\hline Cultural & $\begin{array}{l}\text { Reconocimiento } \\
\text { de las diferentes } \\
\text { etnias que integran } \\
\text { la organización. }\end{array}$ & $\begin{array}{l}\text { Respeto entre los } \\
\text { asociados. } \\
\text { Sistemas de } \\
\text { comunicación. } \\
\text { Integración de los } \\
\text { niños, niñas, jóvenes } \\
\text { y adultos mayores } \\
\text { en las dinámicas } \\
\text { organizacionales. }\end{array}$ & $\begin{array}{l}\text { Ejecución de } \\
\text { prácticas culturales } \\
\text { desde el enfoque } \\
\text { artístico, como } \\
\text { los conocimientos } \\
\text { tradicionales para la } \\
\text { eficiencia productiva. }\end{array}$ & $\begin{array}{l}\text { Preservar } \\
\text { los saberes y } \\
\text { conocimientos } \\
\text { aceptados por los } \\
\text { integrantes de la } \\
\text { organización como } \\
\text { riqueza inmaterial. }\end{array}$ \\
\hline
\end{tabular}

Fuente: Elaboración propia.

\section{Conclusiones}

Las finanzas solidarias permiten una gestión integral como alternativa a las finanzas corporativas, exponiendo su pertinencia y aplicabilidad a organizaciones de base rural. En este sentido, desde los ejes: población, territorio, cultura y economía, junto a la presencia de organizaciones de economía social y solidaria se amplía el espectro de la gestión financiera, social y solidaria de las organizaciones en los contextos rurales que, sin apartarse de los planteamientos de las finanzas corporativas, coexisten, dando prioridad a los objetivos comunitarios. Las bases de gestión financiera identificadas para las organizaciones rurales desde las perspectivas de las finanzas solidarias, ubica a las personas — con sus necesidades y aspiraciones - como pilar de todas las estrategias, procedimientos y decisiones a nivel organizacional, además procura la armonía entre las diferencias culturales y el respeto por el territorio con su medio ambiente, sin desconocer la búsqueda de rendimientos económicos desde lo productivo. La participación colectiva debe estar inserta en todas las etapas de la gestión, para la toma de decisiones, en las acciones a ejecutar, en la verificación, al examinar 
los resultados y aprender de ellos, como en la evaluación permanente a cada acción; ese es el principio fundamental de una gestión financiera desde la perspectiva solidaria, donde todos son responsables y se espera un beneficio común a partir de los relacionamientos sociales y los rendimientos financieros.

\section{Referencias bibliográficas}

Acosta, A. (2013). Otra economía para otra civilización. Revista Temas, No.75, 21-27.

Altadill, M. (03, 2015). Finanzas Solidarias: Herramientas financieras al servicio de causas sociales. Recuperado de: https://repositorio.comillas.edu/rest/bitstreams/5419/ retrieve

Bodie, Z. y Merton, R. (2003). Finanzas. (2a ed.) México D.F: Prentice Hall.

Causse, G. (2012). Islamic Finance: An Alternative Finance or an Antidote to the Crisis of Capitalism? En, W. Barnet y F. Jawadi, Recent Developments in Alternative Finance: Empirical Assessments and Economic Implications (pp. 173-196), Bingley, Reino Unido: Emerald Group Publishing Limited.

Carrizo, A. (2019). Finanzas solidarias con otros. Recuperado de: http://ridaa.unq.edu.ar/ handle/20.500.11807/2899

Chambers, R. (1997). Diagnóstico rural participativo. GTZ, Alemania.

Chao, R. y Prébois, A. (2001). Finanzas solidarias. Recuperado de: http://finsol.socioeco. org/

Chiroque, H. y Niño, L. (2019). La experiencia de la Incubadora Universitaria de Economía, Mercados y Finanzas Solidarias de la Universidad Nacional de Quilmes. Incubación de procesos para impulsar circuitos económicos solidarios. Revista Otra Economía, No. 21 (Vol. 12), pp. 248-260.

Confecámaras - Red de Cámaras de Comercio (2018). Registro Único Empresarial y Social -RUES. Entidades Sin Ánimo de Lucro. Recuperado de: http://www.rues.org.co/ESAL

Contreras, R. (2002), La investigación Acción Participativa (IAP): revisando sus metodologías y sus potencialidades. En, J. Durston y F. Miranda, Experiencias y metodología de la investigación participativa (pp. 9-18), Santiago de Chile: CEPAL.

Coraggio, J. (2013). La economía social y solidaria, y el papel de la economía popular en la estructura económica. Recuperado de: http://www.coraggioeconomia.org/jlc/ archivos\%20para\%20descargar/La\%20economia\%20Popular\%20y\%20Solidaria\%20El\%20 Ser\%20Humano\%20Sobre\%20el\%20Capital.pdf

Coraggio, J. (2011). Economía Social y Solidaria. El trabajo antes que el capital. (1aㅡ. ed.). Quito: Editorial Abya-Yala.

Coraggio, J. (2009). Principios, Instituciones y Prácticas de la Economía Social y Solidaria. Recuperado de: http://www.coraggioeconomia.org/jlc/archivos\%20para\%20descargar/ economiasocial.pdf

Díaz, L. (2016). Sistema de captación de recursos de capital de start up agroindustriales del departamento del Cauca. Caso: Corporación Incubadora de Empresas Agroindustriales del Cauca AGROINNOVA. Tesis para optar el título de Magíster en Administración, Universidad Nacional de Colombia, Palmira, Colombia. 
Ruiz, E. y Oviedo, J. Bases sociales para la configuración de un modelo de gestión financiera...

FAO (2012). Guía metodológica de sistematización. Programa Especial para la Seguridad Alimentaria PESA en Centroamérica. Recuperado de: http://148.202.167.116:8080/ xmlui/bitstream/handle/123456789/2223/Gu\%C3\%ADa\%20metodol\%C3\%B3gica\%20 de $\% 20$ sistematizaci\%C3\%B3n.pdf?sequence $=1$ \&isAllowed $=y$

Forni, P. y De Grande, P. (2020). Triangulación y métodos mixtos en las ciencias sociales contemporáneas. Revista Mexicana de Sociología, No. 1 (Vol. 82), 159-189.

Franco, R. (2014). Fundamentos ontológicos para la construcción del concepto de contametría. Revista Científica General José María Córdova. No. 13 (Vol. 12). pp. 165-190.

Gibson-Graham, J.K., (2007), La construcción de economías comunitarias: las mujeres y la política del lugar. En, W. Harcourt y A. Escobar. Las mujeres y las políticas del lugar (pp. 147-174). México D.F. Universidad Nacional Autónoma de México

Gómez, S. (2000) Organizaciones Rurales en América Latina (marco para su análisis). Revista Austral de Ciencias Sociales, № 4, 27-54.

Guerra, P. (2014). Socioeconomía de la solidaridad. Una teoría para dar cuenta de las experiencias sociales y económicas alternativas (2a ed.). Bogotá: Ediciones Universidad Cooperativa de Colombia.

Guerra, P. (2010). La economía solidaria en Latinoamérica. Recuperado de: https:/base. socioeco.org/docs/la_economia_solidaria_en_latinoamerica_p_guerra.pdf

Jara, O. (1994). Para sistematizar experiencias: una propuesta teórica y práctica (3ª ed.), San José, Costa Rica: ALFORJA.

Landredth, H. y Colander, D. (2006) Historia del pensamiento económico (4⿳亠口冋 ed.). Madrid: McGraw-Hill.

León, L. (2018). Principios de la Economía Popular y Solidaria en el sector asociativo. En, E. Olivares y C. Valladares, Dossier Académico, Río 2018, (pp. 13-20). Guayaquil: CIDE.

Marín, A. (2008). Investigación Cualitativa. Clasificación de la Investigación. Recuperado de: https://metinvestigacion.wordpress.com/2008/03/07/clasificacion-de-la-investigacion/

Max-Neef, M. (1998). Desarrollo a escala humana: conceptos, aplicaciones y algunas reflexiones (2a ed.) Montevideo: Icaria Editorial, S.A.

Mejía. E. (2020). García-Casella: Una vida al servicio de una contabilidad científica y socialmente comprometida. Teuken Bidikay. № 17 (Vol. 11), 21-26.

Nagarajan, G. y Meyer, R. (2005). Finanzas rurales: Avances recientes y lecciones emergentes, debates y oportunidades. Recuperado de: https://www.findevgateway. $\mathrm{org} / \mathrm{sites} / \mathrm{default} / \mathrm{files} /$ publications/files/mfg-es-documento-finanzas-rurales-avancesrecientes-y-lecciones-emergentes-debates-y-oportunidades-7-2005.pdf

Nassar, J. (2019). Finanzas comportamentales: implicaciones de los sesgos de encuadre y cuentas mentales en la toma de decisiones financieras. Tesis para optar el título de Psicólogo, Universidad de los Andes, Bogotá, Colombia.

Ortega, C. (2008). Finanzas Populares y Migración: tejiendo la red para el desarrollo local. Recuperado de: https://www.codespa.org/aprende/publicaciones/finanzas-popularesy-migracion-tejiendo-la-red-para-el-desarrollo-local/

Pastore, R. (2010). Un panorama del resurgimiento de la economía social y solidaria en Argentina. Recuperado de: http://www.unq.edu.ar/advf/documentos/59400be7cff6a.pdf 
Peña, E. (2017). Glosario de desarrollo rural con perspectiva territorial. En C. Corredor, Desarrollo rural territorial, economía campesina y caminos solidarios (pp. 135-209). Popayán, Colombia: Editorial Universidad del Cauca.

Pérez, E.; Maya, D. y Farra, M. (2001). Metodologías participativas en la formulación y planificación de proyectos de desarrollo rural. Fase de diagnóstico en siete municipios del sur del Huila. Cuadernos de desarrollo rural, 47, segundo semestre de 2001. Pontificia Universidad Javeriana, Instituto de Estudios Rurales. Bogotá.

Pérez, M, (2016), Las finanzas alternativas, disciplina al servicio del desarrollo socioeconómico con inclusión. En G. Ziritt, Diálogo de saberes desde las Ciencias Económicas, Administrativas y Contables (pp. 394-406). Sincelejo: Editorial CECAR.

Quiñones, B. y Fernando. S, (2003). El Capital Social en las Finanzas Solidarias. Recuperado de: https://base.socioeco.org/docs/doc-236_es.pdf

Rivas, M. (2013). Índice ICO: Competencias Organizacionales. Diagnóstico Organizacional participativo, rápido y eficiente. Recuperado de: http://www.ard.org.co/midas/ departamentos/agricultores-y-cadenas-de-valor/pdf/Indice_ICO_ultimo.pdf

Ross, S. Westerfield, R. y Jaffe, J. (2012) Finanzas Corporativas (9a ed.), México D.F: McGrawHill.

Saavedra, M. L. y Saavedra, M. J. (2012). Evolución y aportes de la teoría financiera y un panorama de su investigación en México: 2003-2007. Revista Ciencia Administrativa. No. 2. 45-61.

Sanchis, J. y Campos, V. (2018). Economía del Bien Común y Finanzas Éticas. Revista de Economía Pública, Social y Cooperativa, No. 93. 241-264. 